\title{
Retinal Artery Occlusion in a Healthy Pregnant Patient
}

\author{
Yoo-Ri Chung, MD, Jun Bum Kim, MD, Kihwang Lee, MD, Ho Min Lew, MD \\ Department of Ophthalmology, Ajou University School of Medicine, Suwon, Korea
}

\begin{abstract}
Purpose: We report a case of branch retinal artery occlusion (BRAO) in a healthy pregnant woman.
Methods: A 29-year-old pregnant woman presented with decreased vision in her left eye. She had a pale retina with macular edema consistent with BRAO. An extensive workup was performed to determine an etiologic factor. All test results were within normal limits except for her factor VIII activity. Her visual acuity improved from finger counting to 20/30 over 2 months without any treatment.

Results: This case suggests that BRAO can occur in healthy patients without any systemic or ocular disorders.

Conclusions: BRAO can occur in healthy patients without any systemic or ocular disorders, despite an extensile evaluation.
\end{abstract}

Korean Journal of Ophthalmology 22(1):70-71, 2008

Key Words: Factor VIII, Pregnancy, Retinal artery occlusion

Retinal artery occlusions are rare in people younger than 30 years, and most of these patients have some detectable etiologic disorder. ${ }^{1}$ We describe a case of branch retinal artery occlusion (BRAO) in an otherwise healthy pregnant woman.

\section{Case Report}

A 29-year-old Korean woman at 12 weeks' gestation presented with sudden decreased vision in her left eye. Photorefractive keratectomy had been performed on both eyes 10 years earlier, and she had no history of ocular trauma. Her only history of systemic illness involved atopic dermatitis, and she had no problems associated with her pregnancy. On the initial physical examination, she was normotensive and did not complain of headache. Her visual acuity was 20/25 in her right eye, but that in her left eye was reduced to finger counting. The anterior segments and lenses of both eyes were unremarkable. Fundus examination of the left eye showed a pale retina along the inferotemporal vascular arcade associated with diffuse macular edema (Fig. 1), while the right eye was normal. Fluorescence angiography was not performed because she was pregnant.

Laboratory studies included a complete blood count, erythrocyte sedimentation rate, platelet count, prothrombin

Received: August 3, 2007 Accepted: January 16, 2008 Reprint requests to Ho Min Lew, MD. Department of Ophthalmology, Ajou University School of Medicine, San 5 Wonchon-dong, Youngtong-gu, Suwon, 443-721, Korea. Tel: 82-31-219-5260; Fax: 82-31-219-5259, E-mail: hmlew@ajou.ac.kr time, activated prothrombin time, bleeding time, lipid profile, plasma homocysteine, plasma fibrinogen, D-dimer, fibrinogen degradation product, antithrombin III, protein S, protein C, antinuclear antibody, Venereal Disease Research Laboratory (VDRL) assay, and factor V, VII, VIII, and IX assays. Obstetrics and internal medicine consultations were also obtained.

The patient's laboratory workup was unremarkable, except for the level of factor VIII activity, which was 220\% (normal range 60-140\%). Quantitative immunoglobulins were within normal limits, except for the level of immunoglobulin $\mathrm{E}$ detected using a fluoroenzyme-immunometric assay (CAP FEIA), which was $1197 \mathrm{kU} / \mathrm{L}$ (normal range 0-114 kU/L). All other tests were negative.

Her visual acuity improved from finger counting to 20/30 over 2 months, and the improvement of the macular edema was verified by optical coherence tomography.

\section{Discussion}

Retinal artery occlusion is very rare in people younger than 30 years; the average age of patients with retinal artery occlusion is 58.5 years. ${ }^{1,2}$ Retinal artery occlusion is associated with atherosclerosis, hypertension, diabetes, vascular spasm as in migraines, hypercoagulable states, glaucoma, or trauma. ${ }^{3}$ In a study of 27 young patients with retinal artery occlusion reported by Brown et al., all were either Caucasian or African-Americans. ${ }^{1}$ Brown et al. also reported two pregnant patients, each with a history of migraine and one with coexisting increased factor VIII activity. ${ }^{1}$ A case of central retinal artery occlusion was reported with pregnancy 


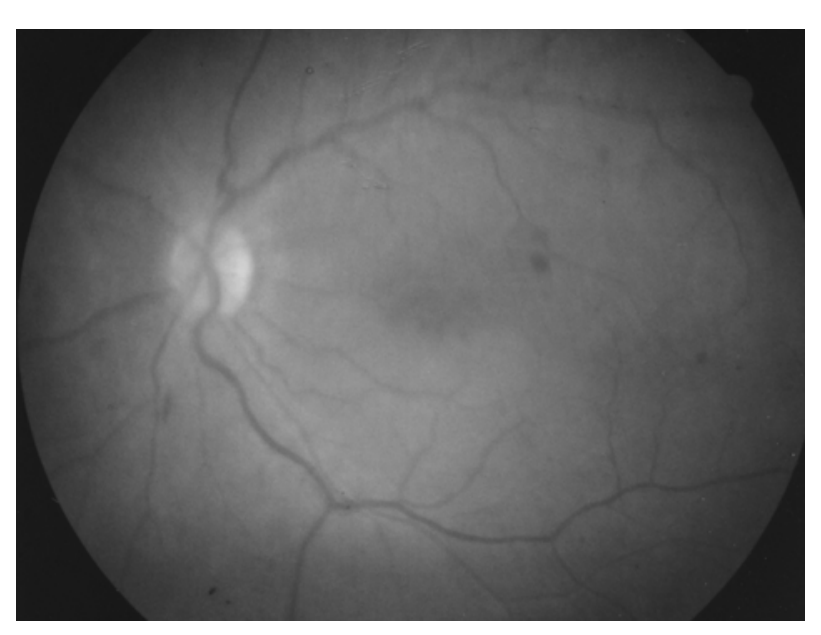

Fig. 1. Funduscopic photograph showing a pale retina with macular edema in the left eye of a 29 -year-old pregnant woman.

as the only known associated factor. ${ }^{3}$ Two cases of pregnant patients with branch retinal arteriolar occlusions have been reported, and these cases were associated with amniotic fluid embolism during abortion or induction of labor., ${ }^{4,5}$ In our patient, who was 29 years old, no evidence of any systemic disorder was revealed on history or in the laboratory investigation.
Increased factor VIII was detected, but the clinical significance of this abnormal result is uncertain because she showed no additional hypercoagulable conditions. Atopic dermatitis was likely the probable cause for the increased immunoglobulin E. To our knowledge, this is the first case of branch retinal artery occlusion in an otherwise healthy pregnant Asian woman. This case suggests that BRAO can occur in healthy patients without any systemic or ocular disorders, despite an extensive evaluation.

\section{References}

1. Brown GC, Magargal LE, Shields JA, Goldberg RE, et al. Retinal arterial obstruction in children and young adults. Ophthalmology 1981;88:18-25.

2. Gull S, Prentice A. Branch retinal artery occlusion in pregnancy. Br J Obstet Gynaecol 1994;101:77-8.

3. LaMonica CB, Foye GJ, Silberman L. A case of sudden retinal artery occlusion and blindness in pregnancy. Obstet Gynecol 1987;69:433-5.

4. Kim IT, Choi JB. Occlusions of branch retinal arterioles following amniotic fluid embolism. Ophthalmologica 2000; 214:305-8.

5. Chang M, Herbert WN. Retinal arteriolar occlusions following amniotic fluid embolism. Ophthalmology 1984; 91:1634-7. 